\title{
The Effect of Heterogeneous Doping on Transport Properties \\ of Lanthanum Dimolybdate
}

\section{Grigoriy S. Partin*,} Irina E. Animitsa and Nadezhda A. Kochetova Ural Federal University named after B.N. Yeltsin 19 Mira Str., Ekaterinburg, 620002, Russia

Received 26.09.2018, received in revised form 10.10.2018, accepted 17.02.2019

\begin{abstract}
A heterogeneous doping method was used for the first time to modify the transport properties of the oxygen-ion conductor $\mathrm{La}(2) \mathrm{Mo}(2) \mathrm{O}(9)$. The effect of temperature and oxygen partial pressure in the gas phase on conductivity of the obtained composite $\left\{0.85 \mathrm{La}_{2} \mathrm{Mo}_{2} \mathrm{O}_{9}-0.15 \mathrm{La}_{2} \mathrm{Mo}_{3} \mathrm{O}_{12}\right\}$ was studied. Introduction of $15 \mathrm{~mol}$. \% an inert low-conductive additional phase La(2)Mo(3)O(12) results in an increase in conductivity of the matrix phase by nearly 1 orders of magnitude. It is associated with appearance of a composite effect. However, there is no suppression of the $\alpha-L a(2) M o(2) O(9) \leftrightarrow \beta-L a(2)$ $M o(2) O(9)$ phase transition. It is shown that the conductivity type of both lanthanum dimolybdate and composite based on it is predominantly ionic in the wide range of oxygen partial pressures.
\end{abstract}

Keywords: lanthanum dimolybdate, LAMOX, heterogeneous doping, composites, oxide-ion conductivity.

Citation: Partin G.S., Animitsa I.E., Kochetova N.A. The effect of heterogeneous doping on transport properties of lanthanum dimolybdate, J. Sib. Fed. Univ. Chem., 2019, 12(2), 221-230. DOI: 10.17516/1998-2836-0120.

(C) Siberian Federal University. All rights reserved

* Corresponding author E-mail address: grigory.partin@urfu.ru 


\title{
Влияние гетерофазного допирования на транспортные свойства димолибдата лантана
}

\author{
Г.С. Партин, И.Е. Анимица, Н.А. Кочетова \\ Уральский федеральныий университет им. Б.Н. Ельиина \\ Россия, 620002, Екатеринбург, ул. Мира, 19
}

Метод гетерогенного допирования впервые использован для модифицирования транспортных свойств кислород-ионного проводника La(2)Mo(2)O(9). Изучено влияние температуры и парииального давления кислорода в газовой фазе на электропроводность композита $\left\{0.85 \mathrm{La}_{2} \mathrm{Mo}_{2} \mathrm{O}_{9}-0.15 \mathrm{La}_{2} \mathrm{Mo}_{3} \mathrm{O}_{12}\right\}$. Введение 15 мол. \% инертной низкопроводящей добавки La(2) Мo(3)О(12) приводит к увеличению проводимости матричной фазы приблизительно на порядок величины, что обусловлено возникновением композиционного эффекта. Однако при этом не происходит подавления фазового перехода $\alpha-L a(2) M o(2) O(9) \leftrightarrow \beta-L a(2) M o(2) O(9)$. Показано, что тип проводимости как димолибдата лантана, так и композита на его основе является преимущественно кислород-ионным в широком диапазоне парицильных давлений кислорода.

Ключевые слова: димолибдат лантана, LAMOX, гетерогенное допирование, композиты, кислород-ионная проводимость.

\section{Введение}

Одним из важнейших направлений развития альтернативной энергетики является разработка топливных элементов, в частности твердооксидных топливных элементов (ТОТЭ), работающих в области средних температур $500-700^{\circ} \mathrm{C}[1,2]$. Получение высокоэффективных материалов для электролитов ТОТЭ, обладающих в заданном температурном диапазоне высокими значениями кислород-ионной или протонной проводимости, - крайне важная материаловедческая задача. На сегодняшний день наиболее эффективной электролитической мембраной выступает стабилизированный оксид циркония (YSZ), однако твердые электролиты на его основе подвержены сильной деградации свойств в ходе эксплуатации ТЭ и имеют высокие рабочие температуры $\sim 900^{\circ} \mathrm{C}$.

В 2000 г. научной группой Лакорре было обнаружено новое семейство соединений LAMOX, обладающих высокой ионной проводимостью. Значения ионной проводимости родоначальника семейства - молибдата лантана $\mathrm{La}_{2} \mathrm{Mo}_{2} \mathrm{O}_{9}$ при $800{ }^{\circ} \mathrm{C}$ достигают $10^{-2} \mathrm{OM}^{-1} \mathrm{~cm}^{-1}$ [3], что сопоставимо с величиной проводимости YSZ при той же температуре. $\mathrm{La}_{2} \mathrm{Mo}_{2} \mathrm{O}_{9}$ существует в двух полиморфных модификациях: $\alpha-\mathrm{La}_{2} \mathrm{Mo}_{2} \mathrm{O}_{9}$ (моноклинная сингония, пространственная группа $P 2_{1}$ ) и $\beta-\mathrm{La}_{2} \mathrm{Mo}_{2} \mathrm{O}_{9}$ (кубическая сингония, пространственная группа $\left.P 2_{1} 3\right)$, причем проводимость $\beta$-модификации на два порядка величины выше проводимости $\alpha$-модификации. Высокие значения проводимости $\beta-\mathrm{La}_{2} \mathrm{Mo}_{2} \mathrm{O}_{9}$ обусловлены особенностями его кристаллической структуры: часть кислородных позиций остается вакантной, что способствует ускоренной миграции ионов кислорода. Фазовый переход $\alpha-\mathrm{La}_{2} \mathrm{Mo}_{2} \mathrm{O}_{9} \leftrightarrow \beta-\mathrm{La}_{2} \mathrm{Mo}_{2} \mathrm{O}_{9}$ 
является обратимым и происходит при температуре $\mathrm{T}_{\text {ф.п. }} \sim 580{ }^{\circ} \mathrm{C}$ [4]. Практическое применение $\mathrm{La}_{2} \mathrm{Mo}_{2} \mathrm{O}_{9}$ в качестве твердого электролита сдерживается из-за недостаточно высоких значений электропроводности ниже температуры фазового перехода и низкой химической устойчивости в восстановительной атмосфере (в низких парциальных давлениях кислорода $\mathrm{Po}_{2}$ ). В целях оптимизации функциональных свойств ионного проводника $\mathrm{La}_{2} \mathrm{Mo}_{2} \mathrm{O}_{9}$ были проведены многочисленные попытки гомогенного допирования [5-12]. Данный метод позволяет увеличить разупорядочение кислородной подрешетки, что, соответственно, способствует облегченной миграции ионов кислорода. В итоге был выявлен круг допантов, способствующих подавлению фазового перехода, стабилизации при комнатной температуре высокопроводящей модификации $\beta-\mathrm{La}_{2} \mathrm{Mo}_{2} \mathrm{O}_{9}$ и увеличению стабильности материала в низких парциальных давлениях кислорода. В работах $[13,14]$ проанализирована совместимость компонентов однокамерных ячеек ТОТЭ с электролитами на основе катион-допированного $\mathrm{La}_{2} \mathrm{Mo}_{2} \mathrm{O}_{9}$ и определены параметры работы таких ячеек. Следует отметить, что способность молибдена к восстановлению в низких $\mathrm{Po}_{2}$ и, соответственно, значительный рост электронной составляющей проводимости [15] делает возможным использование $\mathrm{La}_{2} \mathrm{Mo}_{2} \mathrm{O}_{9}$ в качестве анодов ТОТЭ [16, 17]. Таким образом, в зависимости от природы допирующей добавки материалы на основе $\mathrm{La}_{2} \mathrm{Mo}_{2} \mathrm{O}_{9}$ могут проявлять широкий спектр функциональных свойств (электролиты, смешанные проводники, электродные материалы), соответственно, это позволяет реализовать хорошее механическое и химическое сопряжение компонентов электрохимических устройств. Bсе эти обстоятельства доказывают перспективность систем на основе структуры LAMOX и обусловливают неослабевающий интерес многих исследовательских групп к этим материалам, стимулируя дальнейшее развитие материаловедческого поиска.

В настоящей работе нами впервые использован метод гетерогенного (гетерофазного) допирования с целью увеличения кислород-ионной проводимости $\mathrm{La}_{2} \mathrm{Mo}_{2} \mathrm{O}_{9}$. Данный метод, реализация которого предполагает создание композиционных систем, является менее распространенным способом модифицирования свойств ионных проводников (в частности, на основе сложных оксидов). Хотя этот метод гетерогенного допирования успешно применялся для низкотемпературных протонных проводников - систем «кислая соль (протонный проводник) дисперсный оксид (инертная добавка)» [18], исследования композиционных систем на основе сложных оксидов с кислород-ионной проводимостью продолжают оставаться малочисленными и противоречивыми. Отчасти такая ситуация обусловлена проблемой сопряжения поверхностных атомных сеток двух высокотемпературных матриц.

В данной работе впервые реализован метод гетерогенного допирования димолибдата лантана $\mathrm{La}_{2} \mathrm{Mo}_{2} \mathrm{O}_{9}$, позволяющий получить композиционные системы со значимым уровнем ионной проводимости в области средних температур $\left(500-700{ }^{\circ} \mathrm{C}\right)$. В качестве инертной добавки использовалась соседняя по диаграмме состояния фаза $\mathrm{La}_{2} \mathrm{Mo}_{3} \mathrm{O}_{12}$, обладающая гораздо более низкой проводимостью ( на 2-2.5 порядка величины), чем матричная фаза $\mathrm{La}_{2} \mathrm{Mo}_{2} \mathrm{O}_{9}$. Основная стратегия выбора допирующей фазы - это эвтектический характер взаимодействия в системе $\mathrm{La}_{2} \mathrm{Mo}_{2} \mathrm{O}_{9}-\mathrm{La}_{2} \mathrm{Mo}_{3} \mathrm{O}_{12}$, т. е. отсутствие химического взаимодействия между фазами [19]. Достижение высоких значений ионной проводимости в исследуемых системах связано с так называемым композиционным эффектом, заключающимся в увеличении ионной проводимости солей либо оксидов при их допировании инертным оксидом.

$$
-223-
$$




\section{Экспериментальная часть}

Поликристаллические образцы $\mathrm{La}_{2} \mathrm{Mo}_{2} \mathrm{O}_{9}, \mathrm{La}_{2} \mathrm{Mo}_{3} \mathrm{O}_{12}$, а также композиционный состав $\left\{0.85 \mathrm{La}_{2} \mathrm{Mo}_{2} \mathrm{O}_{9}-0.15 \mathrm{La}_{2} \mathrm{Mo}_{3} \mathrm{O}_{12}\right\}$ (числами обозначены мольные доли компонентов) были получены твердофазным методом из стехиометрических количеств полидисперсных порошков $\mathrm{La}_{2} \mathrm{O}_{3}$ квалификации «х.ч.» и $\mathrm{MoO}_{3}$ квалификации «ос.ч.». Порошки тщательно перетирали в агатовой ступке в среде этилового спирта и проводили три стадии отжига на воздухе со ступенчатым увеличением температуры от 450 до $950{ }^{\circ} \mathrm{C}$ для $\mathrm{La}_{2} \mathrm{Mo}_{2} \mathrm{O}_{9}$ и до $900{ }^{\circ} \mathrm{C}$ для $\mathrm{La}_{2} \mathrm{Mo}_{3} \mathrm{O}_{12}$ с изотермическими выдержками 18-20 ч и промежуточными перетираниями. Композит подвергали термообработке при $900{ }^{\circ} \mathrm{C}$ в течение 24 ч. Фазовый состав полученных керамических образцов установлен методом рентгенофазового анализа (РФА) с использованием дифрактометра Bruker D8 Advance (Германия) в $\mathrm{CuK}_{\alpha}$-излучении с шагом $0.02^{\circ}$ в интервале углов $2 \theta=10-80^{\circ}$, съемка велась при комнатной температуре. Обработку дифракционных данных проводили с применением полнопрофильного анализа поликристаллических веществ по методу Ритвельда [20] в программе FullProf.

Брикеты для измерения электропроводности в форме дисков толщиной $2-3$ мм и диаметром $\sim 15$ мм получали одноосным прессованием порошков на ручном гидравлическом прессе в текстолитовой пресс-форме при давлении 8 МПа с последующим отжигом при 900-950 으 в течение суток. В качестве электродов использовалась паста, состоящая из порошка платины и спиртового раствора канифоли, которую наносили на шлифованные торцевые поверхности спеченных брикетов. Вжигание электродов проводили при медленном (1-2 $\left.{ }^{\circ} \mathrm{C} / \mathrm{мин}\right)$ нагреве до $900{ }^{\circ} \mathrm{C}$ с последующей 6-часовой выдержкой.

Электропроводность была измерена методом импедансной спектроскопии на переменном токе в диапазоне частот 500 Гц-3 МГц при помощи импедансметра Elins Impedancemeter Z-1000Р (Россия). Измерения проводили в температурном интервале $900-300{ }^{\circ} \mathrm{C}$ со скоростью охлаждения $1{ }^{\circ} \mathrm{C} /$ мин, спектры импеданса записывались на персональный компьютер каждые 25 мин. Оценку объемного и зернограничного вкладов в общую проводимость осуществляли с использованием метода эквивалентных схем в программе ZView.

Парциальное давление кислорода $\mathrm{Po}_{2}$ в диапазоне 0.21-3.2×10-5 атм задавали и поддерживали посредством кислородного электрохимического насоса под управлением автоматического регулятора Zirconia-M [21].

\section{Результаты и обсуждение}

Структура $\mathrm{La}_{2} \mathrm{Mo}_{2} \mathrm{O}_{9}$, согласно данным РФА (рис. $1 a$ ), соответствует моноклинной $P 2_{1}$ сверхструктуре $\alpha$-модификации (уточненные параметры ячейки: $a=7.146(0) \AA, b=7.145(8) \AA$, $\left.c=7.160(2) \AA, \beta=89.49(9){ }^{\circ}\right)$, которая описана в работе [3]. Рентгенограмма тримолибдата лантана $\mathrm{La}_{2} \mathrm{Mo}_{3} \mathrm{O}_{12}$ представлена на рис. 16. $\mathrm{La}_{2} \mathrm{Mo}_{3} \mathrm{O}_{12}$ характеризуется моноклинной структурой с пространственной группой $C 2 / c$ (уточненные параметры ячейки: $a=17.009(3) \AA, b=11.944(8) \AA$, $\left.c=16.058(5) \AA, \beta=108.49(9)^{\circ}\right)$, что хорошо согласуется с данными работы [22]. Рентгенограмма композиционной системы $\left\{0.85 \mathrm{La}_{2} \mathrm{Mo}_{2} \mathrm{O}_{9}-0.15 \mathrm{La}_{2} \mathrm{Mo}_{3} \mathrm{O}_{12}\right\}$ представлена на рис. 18: уточненные параметры ячеек компонентов, входящих в состав композита, практически идентичны параметрам ячеек индивидуальных фаз (рис. $1 a$, $\sigma$ ), что свидетельствует об отсутствии химического взаимодействия между компонентами. 

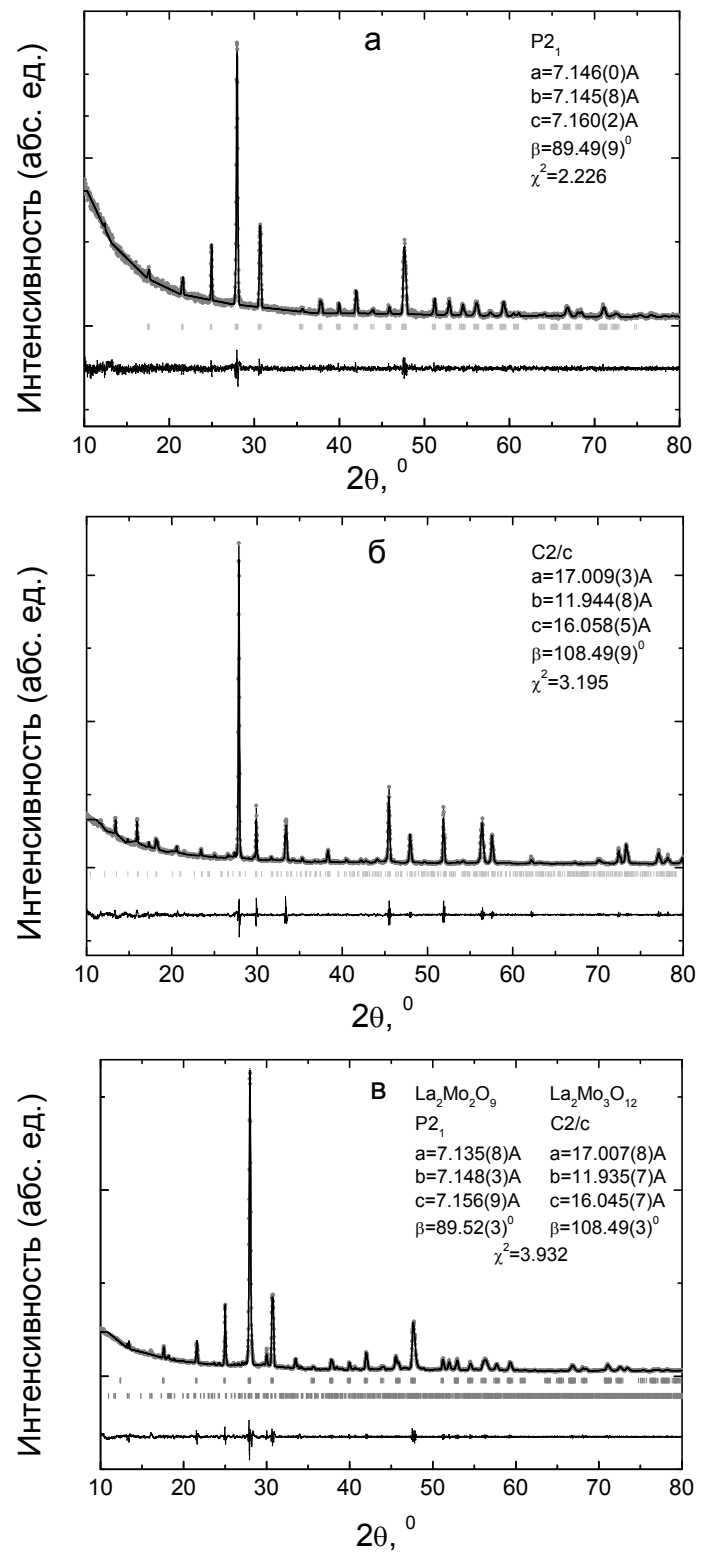

Рис. 1. Рентгенограммы порошкообразных образцов: a - $\mathrm{La}_{2} \mathrm{Mo}_{2} \mathrm{O}_{9} ; \sigma-\mathrm{La}_{2} \mathrm{Mo}_{3} \mathrm{O}_{12} ;$ в - $\left\{0.85 \mathrm{La}_{2} \mathrm{Mo}_{2} \mathrm{O}_{9}-\right.$ $\left.0.15 \mathrm{La}_{2} \mathrm{Mo}_{3} \mathrm{O}_{12}\right\}$ (черные кривые - рассчитанные, серые точки - экспериментальные, черные кривые в нижней части рисунка - разностные рентгеновские профили, серые риски соответствуют угловым положениям рефлексов)

Fig. 1. X-ray diffraction patterns of powder samples: a $-\mathrm{La}_{2} \mathrm{Mo}_{2} \mathrm{O}_{9} ; \sigma-\mathrm{La}_{2} \mathrm{Mo}_{3} \mathrm{O}_{12} ; \mathbf{B}-\left\{0.85 \mathrm{La}_{2} \mathrm{Mo}_{2} \mathrm{O}_{9}-\right.$ $\left.0.15 \mathrm{La}_{2} \mathrm{Mo}_{3} \mathrm{O}_{12}\right\}$ (the black curves - calculated, the gray dots - observed, the black curves at the bottom of figure difference $\mathrm{x}$-ray profiles, the gray vertical tics corresponds to Bragg positions of peaks)

Измерения сопротивления, выполненные методом импедансной спектроскопии, позволили выделить вклад объема и границ зерен из общего сопротивления образца. На рис. 2 приведены примеры обработки спектров импеданса, на которых, в общем случае, можно выделить два полукруга, соответствующих объемному и зернограничному сопротивлениям. На спектрах 

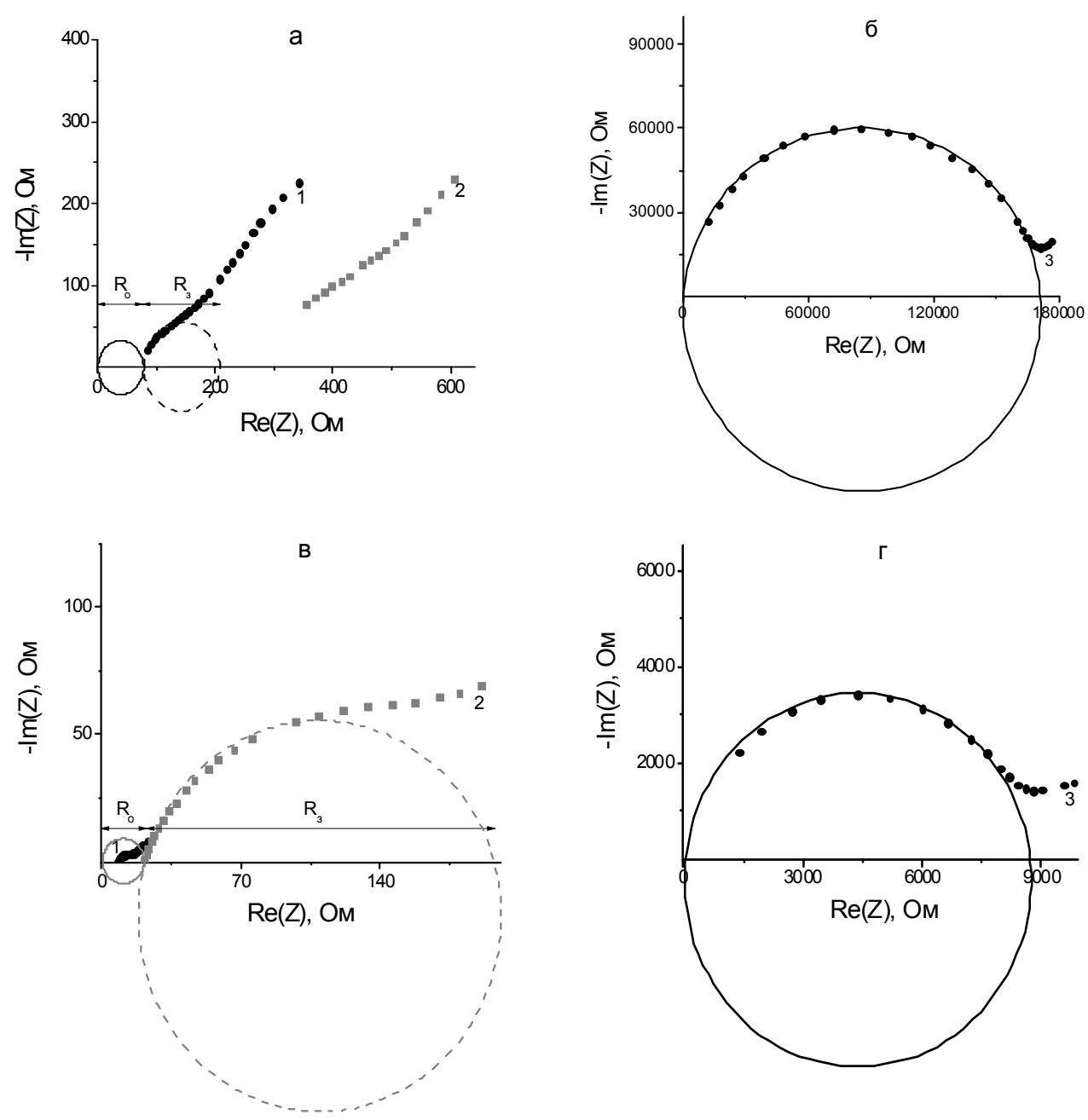

Рис. 2. Примеры спектров импеданса образцов: а, б- $\mathrm{La}_{2} \mathrm{Mo}_{2} \mathrm{O}_{9} ;$ в, г - $\left\{0.85 \mathrm{La}_{2} \mathrm{Mo}_{2} \mathrm{O}_{9}-0.15 \mathrm{La}_{2} \mathrm{Mo}_{3} \mathrm{O}_{12}\right\}$, измеренных на воздухе при: $1-800^{\circ} \mathrm{C} ; 2-600^{\circ} \mathrm{C} ; 3-400^{\circ} \mathrm{C}$. Сплошными кругами выделено сопротивление объема $\mathrm{R}_{\mathrm{o}}$, пунктирными кругами - сопротивление границ зерен $\mathrm{R}_{3}$

Fig. 2. Examples of impedance spectra measured in air for samples: a, $\sigma-\mathrm{La}_{2} \mathrm{Mo}_{2} \mathrm{O}_{9}, \mathrm{~B}, \Gamma-\left\{0.85 \mathrm{La}_{2} \mathrm{Mo}_{2} \mathrm{O}_{9}-\right.$ $\left.0.15 \mathrm{La}_{2} \mathrm{Mo}_{3} \mathrm{O}_{12}\right\}$ at: $1-800{ }^{\circ} \mathrm{C} ; 2-600{ }^{\circ} \mathrm{C} ; 3-400{ }^{\circ} \mathrm{C}$. The solid circles indicate bulk resistance $\mathrm{R}_{\mathrm{b}}$, the dash circles indicate grain boundary resistance $\mathrm{R}_{\mathrm{gb}}$

импеданса, относящихся к высокотемпературной области (рис. $2 a, 8$ ), хорошо различимы две полуокружности - сопротивление объема зерна и сопротивление границ зерен, причем в области низких частот вторая полуокружность пересекается с лучом, соответствующим электродному отклику; на спектрах, полученных при $\mathrm{T}<\mathrm{T}_{\text {ф.л. }}$ (рис. 26 , г), наблюдается одна полуокружность, соответствующая объемному сопротивлению.

На рис. 3 показаны политермы объемной электропроводности, полученные на воздухе, для фаз $\mathrm{La}_{2} \mathrm{Mo}_{2} \mathrm{O}_{9}$ и $\mathrm{La}_{2} \mathrm{Mo}_{3} \mathrm{O}_{12}$, а также композита $\left\{0.85 \mathrm{La}_{2} \mathrm{Mo}_{2} \mathrm{O}_{9}-0.15 \mathrm{La}_{2} \mathrm{Mo}_{3} \mathrm{O}_{12}\right\}$. Политермы проводимостей $\mathrm{La}_{2} \mathrm{Mo}_{2} \mathrm{O}_{9}$ и композита на его основе имеют схожий характер, добавление инертной фазы $\mathrm{La}_{2} \mathrm{Mo}_{3} \mathrm{O}_{12}$ не приводит к подавлению фазового перехода, выраженного на рис. 3 скачкообразным увеличением проводимости в температурном интервале $530-580{ }^{\circ} \mathrm{C}$, и стабилиза- 


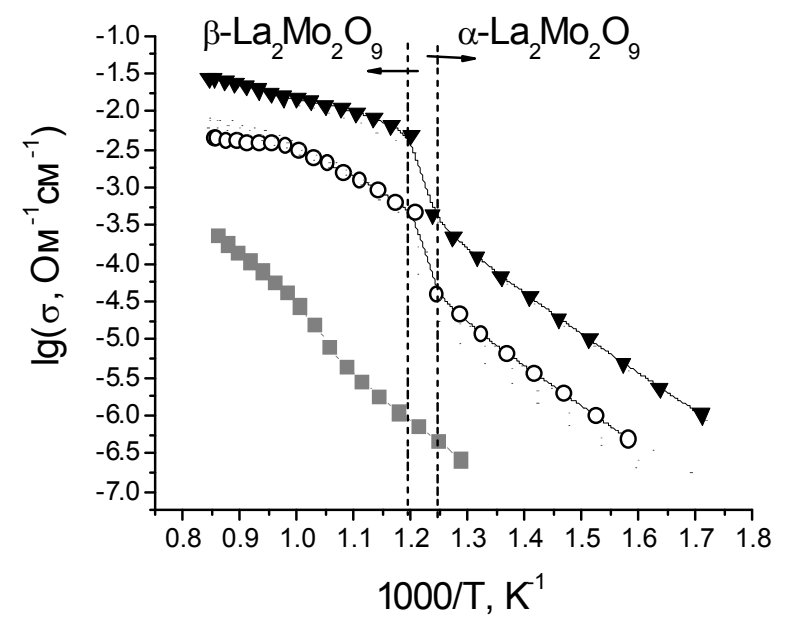

Рис. 3. Температурные зависимости объемной электропроводности, полученные на воздухе, для образцов: ○ $-\mathrm{La}_{2} \mathrm{Mo}_{2} \mathrm{O}_{9} ;-\mathrm{La}_{2} \mathrm{Mo}_{3} \mathrm{O}_{12} ; \boldsymbol{\nabla}-\left\{0.85 \mathrm{La}_{2} \mathrm{Mo}_{2} \mathrm{O}_{9}-0.15 \mathrm{La}_{2} \mathrm{Mo}_{3} \mathrm{O}_{12}\right\}$. Пунктирными линиями выделен температурный интервал, соответствующий фазовому переходу $\alpha-\mathrm{La}_{2} \mathrm{Mo}_{2} \mathrm{O}_{9} \leftrightarrow \beta-\mathrm{La}_{2} \mathrm{Mo}_{2} \mathrm{O}_{9}$. Стрелками указаны температурные области существования $\alpha$ - и $\beta$-модификаций

Fig. 3. Temperature dependences of bulk conductivity measured in air for samples: $\bigcirc-\mathrm{La}_{2} \mathrm{Mo}_{2} \mathrm{O}_{9}$; $-\mathrm{La}_{2} \mathrm{Mo}_{3} \mathrm{O}_{12}$; $\boldsymbol{\nabla}-\left\{0.85 \mathrm{La}_{2} \mathrm{Mo}_{2} \mathrm{O}_{9}-0.15 \mathrm{La}_{2} \mathrm{Mo}_{3} \mathrm{O}_{12}\right\}$. The dashed lines indicate temperature range corresponding to the phase transition $\alpha-\mathrm{La}_{2} \mathrm{Mo}_{2} \mathrm{O}_{9} \leftrightarrow \beta-\mathrm{La}_{2} \mathrm{Mo}_{2} \mathrm{O}_{9}$. The arrows indicate temperature regions of existence of $\alpha$ - and $\beta$-modifications

ции высокопроводящей $\beta$-модификации при комнатной температуре. Как видно, происходит увеличение объемной проводимости композита примерно на порядок величины относительно проводимости матричной фазы $\mathrm{La}_{2} \mathrm{Mo}_{2} \mathrm{O}_{9}$, что свидетельствует о наличии композиционного эффекта. При этом эффект увеличения проводимости композита наблюдался во всей исследованной температурной области, т. е. как для $\alpha$-, так и для $\beta$-модификаций $\mathrm{La}_{2} \mathrm{Mo}_{2} \mathrm{O}_{9}$. Проводимость фазы $\mathrm{La}_{2} \mathrm{Mo}_{3} \mathrm{O}_{12}$ низкая и примерно на 2-2.5 порядка ниже $\mathrm{La}_{2} \mathrm{Mo}_{2} \mathrm{O}_{9}$. Рассчитанные из температурных зависимостей значения энергий активации $\mathrm{E}_{\mathrm{a}}$ для $\mathrm{La}_{2} \mathrm{Mo}_{2} \mathrm{O}_{9}$ равны 1.18 эВ и 0.62 эВ в низко- и в высокотемпературной области, соответственно, для $\alpha$ - и $\beta$-фазы, что хорошо согласуется с литературными данными $[23,24]$. Для композита $\left\{0.85 \mathrm{La}_{2} \mathrm{Mo}_{2} \mathrm{O}_{9}-0.15 \mathrm{La}_{2} \mathrm{Mo}_{3} \mathrm{O}_{12}\right\}$ соответствующие энергии активации равны 1.13 эВ и 0.50 эВ, т. е. наблюдается тенденция снижения величины $\mathrm{E}_{\mathrm{a}}$.

Таким образом, эффект увеличения проводимости $\mathrm{La}_{2} \mathrm{Mo}_{2} \mathrm{O}_{9}$ при допировании низкопроводящей фазой $\mathrm{La}_{2} \mathrm{Mo}_{3} \mathrm{O}_{12}$ не связан с подавлением фазового $\alpha$ - $\beta$-перехода, на настоящий момент не удалось объяснить механизм данного эффекта, так как необходимо дальнейшее изучение широких концентрационных областей с привлечением методов исследования морфологии поверхности. Однако уже можно сделать вывод о перспективности как самой композиционной системы, так и данного метода модифицирования свойств ионных проводников.

Что касается эволюции зернограничной составляющей проводимости, то можно отметить прирост доли зернограничного вклада в композите при снижении температуры (рис. 2). Соотношение объемного и зернограничного вкладов в матричной фазе изменяется не очень значительно. 

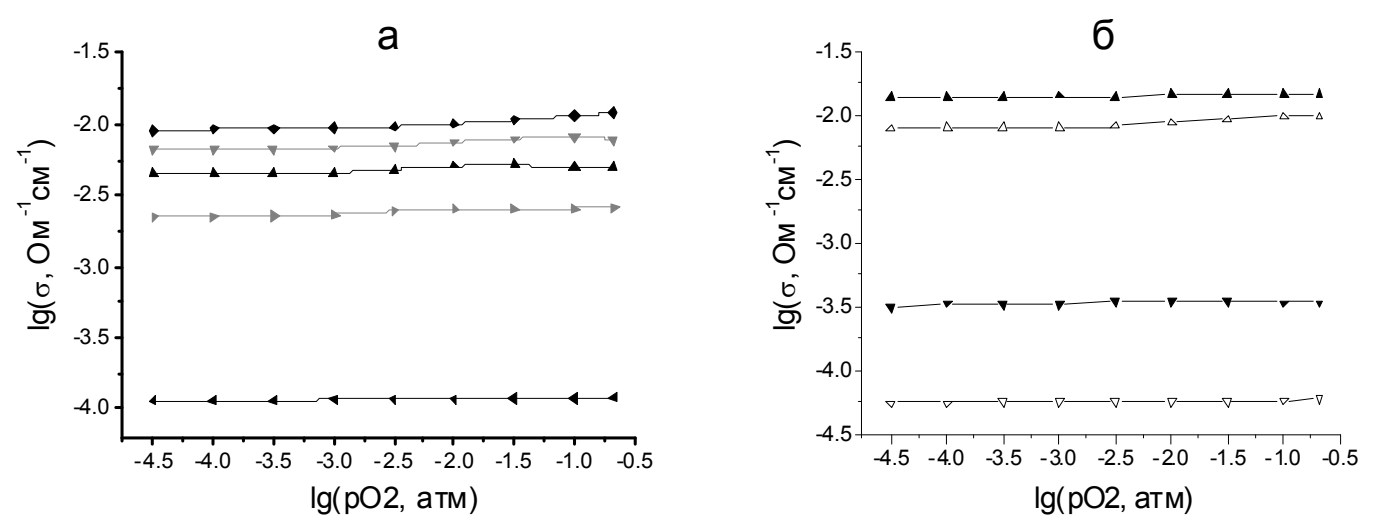

Рис. 4. Зависимости электропроводности от парциального давления кислорода $\mathrm{Po}_{2}$ для а $-\mathrm{La}_{2} \mathrm{Mo}_{2} \mathrm{O}_{9}$ при температурах: $-830{ }^{\circ} \mathrm{C} ; \nabla-780{ }^{\circ} \mathrm{C} ; \boldsymbol{\Delta}-730{ }^{\circ} \mathrm{C} ;-630{ }^{\circ} \mathrm{C} ;-530{ }^{\circ} \mathrm{C}$; для б - $\left\{0.85 \mathrm{La}_{2} \mathrm{Mo}_{2} \mathrm{O}_{9}-\right.$ $\left.0.15 \mathrm{La}_{2} \mathrm{Mo}_{3} \mathrm{O}_{12}\right\}$ при температурах: $\boldsymbol{\Delta}-800{ }^{\circ} \mathrm{C} ; \boldsymbol{\nabla}-500{ }^{\circ} \mathrm{C}$ (открытые значки - $\mathrm{La}_{2} \mathrm{Mo}_{2} \mathrm{O}_{9}$; закрытые значки - $\left.\left\{0.85 \mathrm{La}_{2} \mathrm{Mo}_{2} \mathrm{O}_{9}-0.15 \mathrm{La}_{2} \mathrm{Mo}_{3} \mathrm{O}_{12}\right\}\right)$

Fig. 4. Dependences of conductivity on oxygen partial pressure $\mathrm{Po}_{2}$ for a $-\mathrm{La}_{2} \mathrm{Mo}_{2} \mathrm{O}_{9}$ at temperatures: $-830{ }^{\circ} \mathrm{C}$; $\nabla-780{ }^{\circ} \mathrm{C} ; \boldsymbol{\Delta}-730{ }^{\circ} \mathrm{C} ;-630{ }^{\circ} \mathrm{C} ;-530{ }^{\circ} \mathrm{C}$; for $\sigma-\left\{0.85 \mathrm{La}_{2} \mathrm{Mo}_{2} \mathrm{O}_{9}-0.15 \mathrm{La}_{2} \mathrm{Mo}_{3} \mathrm{O}_{12}\right\}$ at temperatures: $\boldsymbol{\Delta}-$ $800{ }^{\circ} \mathrm{C} ; \boldsymbol{\nabla}-500{ }^{\circ} \mathrm{C}$ (the open signs $-\mathrm{La}_{2} \mathrm{Mo}_{2} \mathrm{O}_{9}$; the closed signs $-\left\{0.85 \mathrm{La}_{2} \mathrm{Mo}_{2} \mathrm{O}_{9}-0.15 \mathrm{La}_{2} \mathrm{Mo}_{3} \mathrm{O}_{12}\right\}$ )

В ранних работах $[5,15]$ сообщалось, что основными носителями заряда в $\mathrm{La}_{2} \mathrm{Mo}_{2} \mathrm{O}_{9}$ являются ионы кислорода $\mathrm{O}^{2-}$. В данном исследовании преобладание ионных носителей заряда доказывали посредством измерения электропроводности при вариации парциального давления кислорода в газовой фазе. Экспериментальные изотермы, представленные на рис. 4, имеют вид, близкий к линейному, и относятся к электролитической области. Согласно теоретическим представлениям о влиянии $\mathrm{Po}_{2}$ на концентрацию дефектов в кристаллической решетке [25], $\sigma_{\text {ion }}$ в средней электролитической области не зависит от величины парциального давления, а $\sigma_{\mathrm{e}, \mathrm{h}} \sim \mathrm{Po}_{2}{ }^{ \pm 1 / m}$, где $\sigma_{\mathrm{ion}}, \sigma_{\mathrm{h}}, \sigma_{\mathrm{e}}-$ ионная, дырочная и электронная проводимость соответственно, а знак и значение $1 / \mathrm{m}$ зависят от природы электронных носителей и типа разупорядочения кристаллической решетки. Как видим на рис. $4 a$ и 46 , величина проводимости практически не меняется при снижении $\mathrm{Po}_{2}$, показатель $1 / \mathrm{m}$ близок к нулю, поэтому можно сделать вывод о доминирующем ионном типе проводимости, сохраняющемся как для матричной фазы $\mathrm{La}_{2} \mathrm{Mo}_{2} \mathrm{O}_{9}$, так и для композита $\left\{0.85 \mathrm{La}_{2} \mathrm{Mo}_{2} \mathrm{O}_{9}-0.15 \mathrm{La}_{2} \mathrm{Mo}_{3} \mathrm{O}_{12}\right\}$ в широком интервале парциальных давлений кислорода $0.21-3.2 \times 10^{-5}$ атм. Ионные числа переноса $\mathrm{t}_{\text {ion }}$, рассчитанные из зависимостей электропроводности от $\mathrm{Po}_{2}$ по формуле $\mathrm{t}_{\text {ion }}=\sigma_{\text {ion }} /\left(\sigma_{\text {ion }}+\sigma_{\mathrm{h}}\right)$, близки к единице.

\section{Выводы}

Композиционный состав $\left\{0.85 \mathrm{La}_{2} \mathrm{Mo}_{2} \mathrm{O}_{9}-0.15 \mathrm{La}_{2} \mathrm{Mo}_{3} \mathrm{O}_{12}\right\}$ на основе фаз семейства LAMOX, как и его компоненты, был получен твердофазным методом, отсутствие между фазами композита химического взаимодействия подтверждено рентгенографически.

Был отмечен рост объемной составляющей проводимости композита $\left\{0.85 \mathrm{La}_{2} \mathrm{Mo}_{2} \mathrm{O}_{9}-\right.$ $\left.0.15 \mathrm{La}_{2} \mathrm{Mo}_{3} \mathrm{O}_{12}\right\}$ относительно проводимости матричной фазы $\mathrm{La}_{2} \mathrm{Mo}_{2} \mathrm{O}_{9}$ во всем исследованном температурном диапазоне. Проводимость композита увеличилась приблизительно на 
порядок величины, что указывает на наличие композиционного эффекта в исследуемой системе. Влияние гетерогенного допанта $\mathrm{La}_{2} \mathrm{Mo}_{3} \mathrm{O}_{12}$ на электрические свойства композита более выражено при температурах ниже $\mathrm{T}_{\phi . . .} \sim 580{ }^{\circ} \mathrm{C}$, однако следует отметить, что инертная добавка не способствует подавлению фазового перехода и полной стабилизации при комнатной температуре высокопроводящей $\beta$-модификации $\mathrm{La}_{2} \mathrm{Mo}_{2} \mathrm{O}_{9}$. Вклад ионных носителей заряда в общую проводимость остается доминирующим как для $\mathrm{La}_{2} \mathrm{Mo}_{2} \mathrm{O}_{9}$, так и для композита в исследованном интервале $\mathrm{Po}_{2} 0.21-3.2 \times 10^{-5}$ атм. Рассчитанные ионные числа переноса $t_{\text {ion }}$ близки к единице.

\section{Благодарности}

Работа выполнена в рамках государственного задания Министерства образования и науки Российской Федерации № 4.2288.2017/4.6.

\section{Список литературы}

1. Malavasi L., Fisher C.A.J., Islam M.S. Oxide-ion and proton conducting electrolyte materials for clean energy applications: structural and mechanistic features. Chem. Soc. Rev. 2010. Vol. 39, P. 4370-4387.

2. Singh B., Ghosh S., Aich S., Roy B. Low temperature solid oxide electrolytes (LT-SOE): A review. Journal of Power Sources 2017. Vol. 339, P. 103-135.

3. Goutenoire F., Isnard O., Retoux R., Lacorre P. Crystal structure of $\mathrm{La}_{2} \mathrm{Mo}_{2} \mathrm{O}_{9}$, a new fast oxide-ion conductor. Chem. Mater 2000. Vol. 12, P. 2575-2580.

4. Malavasi L., Kim H.J., Billinge S.J.L., Proffen T., Tealdi C., Flor G. Nature of the monoclinic to cubic phase transition in the fast oxygen ion conductor $\mathrm{La}_{2} \mathrm{Mo}_{2} \mathrm{O}_{9}$ (LAMOX). J. Am. Chem. Soc. 2007. Vol. 129, P. 6903-6907.

5. Collado J.A., Aranda M.A.G., Cabeza A., Olivera-Pastor P., and Bruque S. Synthesis, structures, and thermal expansion of the $\mathrm{La}_{2} \mathrm{~W}_{2-\mathrm{x}} \mathrm{Mo}_{\mathrm{x}} \mathrm{O}_{9}$ series. Journal of Solid State Chemistry. 2002. Vol. 167, P. 80-85.

6. Marozau I.P., Marrero-Lopez D., Shaula A.L., Kharton V.V., Tsipis E.V., Nunez P., Frade J.R. Ionic and electronic transport in stabilized $\beta-\mathrm{La}_{2} \mathrm{Mo}_{2} \mathrm{O}_{9}$ electrolytes. Electrochimica Acta. 2004. Vol. 49, P. 3517-3524.

7. Tealdi C., Chiodelli G., Malavasi L., and Flor G. Effect of alkaline-doping on the properties of $\mathrm{La}_{2} \mathrm{Mo}_{2} \mathrm{O}_{9}$ fast oxygen ion conductor. J. Mater. Chem. 2004. Vol. 14, P. 3553-3557.

8. Subramania A., Saradha T., Muzhumathi S. Synthesis, sinterability and ionic conductivity of nanocrystalline Pr-doped $\mathrm{La}_{2} \mathrm{Mo}_{2} \mathrm{O}_{9}$ fast oxide-ion conductors. Journal of Power Sources. 2007. Vol. 167, P. 319-324.

9. Marrero-Lopez D., Pena-Martinez J., Ruiz-Morales J.C., Perez-Coll D., Martin-Sedeno M.C., Nunez P. Structural and electrical characterisation of $\mathrm{Nb}^{5+}$ and $\mathrm{Cr}^{6+}$ substituted $\mathrm{La}_{2} \mathrm{Mo}_{2} \mathrm{O}_{9}$. Bol. Soc. Esp. Ceram. 2008. Vol. 47, P. 213-218.

10. Ruan B., Yan B., Zhang J. Effect of oxygen partial pressure on the electrical conductivity of $\mathrm{La}_{2} \mathrm{Mo}_{2-\mathrm{x}} \mathrm{W}_{\mathrm{x}} \mathrm{O}_{9-\delta}(\mathrm{x}=0.0,0.5,0.7)$ materials. Solid State Sciences. 2012. Vol. 14, P. 840-848.

11. Basu S., Devi P.S., Maiti H.S., Bandyopadhyay N.R. Synthesis, thermal and electrical analysis of alkaline earth doped lanthanum molybdate. Solid State Ionics. 2013. Vol. 231, P. 87-93. 
12. Le M.-V., Tsai D.-S., Yao C.-C., Lo J.-C., Giao T.P. Properties of $10 \%$ Dy-doped $\mathrm{La}_{2} \mathrm{Mo}_{2} \mathrm{O}_{9}$ and its electrolyte performance in single chamber solid oxide fuel cell. Journal of Alloys and Compounds. 2014. Vol. 582, P. 780-785.

13. Marrero-Lopez D., Pena-Martinez J., Ruiz-Morales J.C., Perez-Coll D., Martin-Sedeno M.C., Nunez P. Applicability of $\mathrm{La}_{2} \mathrm{Mo}_{2-\mathrm{y}} \mathrm{W}_{\mathrm{y}} \mathrm{O}_{9}$ materials as solid electrolyte for SOFCs. Solid State Ionics. 2007. Vol. 178, P. 1366-1378.

14. Le M.-V., Tsai D.-S., Nguyen T.-A. BSCF/GDC as a refined cathode to the single-chamber solid oxide fuell cell based on a LAMOX electrolyte. Ceramics International. 2018. Vol. 44, P. 17261730.

15. Marrero-Lopez D., Ruiz-Morales J.C., Perez-Coll D., Nunez P., Abrantes J.C.C., Frade J.R. Stability and transport properties of $\mathrm{La}_{2} \mathrm{Mo}_{2} \mathrm{O}_{9}$. J. Solid State Electrochem. 2004. Vol. 8, P. 638-643.

16. Buvat G., Quarez E., Joubert O.J. Innovative solid oxide fuel cells based on $\mathrm{BaIn}_{0.3} \mathrm{Ti}_{0.7} \mathrm{O}_{2.85}$ electrolyte and $\mathrm{La}_{2} \mathrm{Mo}_{2} \mathrm{O}_{9}$ amorphous reduced phase as anode material. Journal of Power Sources. 2016. Vol. 302, P. 107-113.

17. Buvat G., Sellemi H., Ravella U.K., Barre M., Coste S., Corbel G., Lacorre P. Reduction kinetics of $\mathrm{La}_{2} \mathrm{Mo}_{2} \mathrm{O}_{9}$ and phase evolution during reduction and reoxidation. Inorg. Chem. 2016. Vol. 55, P. 2522-2533.

18. Lavrova G.V., Shutova E.S., Ponomareva V.G., and Dunyushkina L.A. Proton conductivity and interphase interaction in $\mathrm{CsH}_{2} \mathrm{PO}_{4}-\mathrm{SrZrO}_{3}$ composites. Russian Journal of Electrochemistry. 2013. Vol. 49, P. 718-724.

19. Роде Е.Я., Лысанова Г.В., Гохман Л.З. Диаграммы состояния систем, образованных окислами РЗЭ и трехокисью молибдена. Неорг. материалы. 1971. Т. 7, С. 2101-2103. [Rode E.Ya., Lysanova G.V., Gokhman L.Z. Phase Diagram of $\mathrm{Ln}_{2} \mathrm{O}_{3}-\mathrm{MoO} 3$ systems. Inorg. Mater. 1971. Vol. 7, P. 2101-2103. (In Russ.)]

20. Reitveld H. A profile refinement method for nuclear and magnetic structures. Journal of Applied Crystallography. 1969. Vol. 2, P. 65-71.

21. Пат. 2395832 РФ. Удилов А.Е., Вылков А.И. Способ поддержания заданного давления кислорода. Опубл. 27.07.2010. [Pat. 2395832. RU. Udilov A.E., Vylkov A.I. Method to maintain preset pressure of oxygen. Publ. Date 27.07.2010 (In Russ.)]

22. Jeitschko W. Crystal structure of $\mathrm{La}_{2}\left(\mathrm{MoO}_{4}\right)_{3}$, a new ordered defect scheelite type. Acta Cryst. 1973. Vol. B29, P. 2074-2081.

23. Peet J.R., Fuller C.A., Frick B., Zbiri M., Piovano A., Johnson M.R., Radosavljevic Evans I. Direct observation of oxide ion dynamics in $\mathrm{La}_{2} \mathrm{Mo}_{2} \mathrm{O}_{9}$ on the nanosecond timescale. Chemistry of materials. 2017. Vol. 29, P. 3020-3028.

24. Liu J., Chater R.J., Hagenhoff B., Morris R.J.H., Skinner S.J. Surface Enhancement of Oxygen Exchange and Diffusion in the Ionic Conductor $\mathrm{La}_{2} \mathrm{Mo}_{2} \mathrm{O}_{9}$. Solid State Ionics. 2010. Vol. 181, P. 812818.

25. Чеботин В.Н., Перфильев М.В. Электрохимия твердых электролитов. М.: Химия, 1978. 310 c. [Chebotin V.N., Perfil’ev M.V. Electrochemistry of solid electrolytes. Moscow: Khimiya, 1978. 310 p. (In Russ.)] 